\title{
Review on gas-liquid mixing analysis in multiscale stirred vessel using CFD
}

\author{
Baharak Sajjadi, Abdul Aziz Abdul Raman *, Shaliza \\ Ibrahim and Raja Shazrin Shah Raja Ehsan Shah \\ Faculty of Engineering, Department of Chemical \\ Engineering, University of Malaya, 50603 Kuala Lumpur, \\ Malaysia, e-mail: azizraman@um.edu.my
}

\section{Introduction}

Stirred vessels are widely used in the chemical, biochemical,

mineral, pharmaceutical, and biotechnological industries to

perform chemical operations such as halogenation, hydrogenation,

oxidation, crystallization, polymerization, and biological

fermentation (Van den Akker 2006, Buffo et al. 2012 ).

Generally, mixing affects around $25 \%$ of all process industry

operations (Yeoh et al. 2004 ). The primary aim of mixing

is to increase the interfacial mass transfer rate in these vessels,

which is related to the interfacial area between phases,

volume fraction hold-up, bubble or droplet or particle size

distribution, dynamic equilibrium between coalescence and

breakage rate, turbulence level, fl uid properties of dispersed

and continuous phases, and mechanical mixing parameters

such as impeller size and model, vessel size, and fl ow velocity

(Bouuyatiotis and Thonton 1967 ). As it is impossible to

cover all the mixing process parameters experimentally, computational

fl uid dynamics (CFD) has created opportunities to

visualize the mixing phenomena (Haresh et al. 2010 ). CFD is

able to predict fl uid fl ows, chemical reaction rates, mass and

heat transfer rates, and other occurrences by solving a set of

appropriate mathematical equations (Ding et al. 2010 ). CFD

also provides useful information for regions with intense or

mild turbulence zones, Reynolds stresses, vortex structures,

circulation patterns, fl ow behavior, and many other parameters

(Haresh et al. 2010 ). Notwithstanding its potential, fundamental

knowledge in multiphase fl ow simulations is still 
lacking because of its complexities, particularly in stirred vessels that have movable pieces. For example, CFD is still undeveloped to assess the non-homogeneity impact and phase property changes in stirred polymerization reactors (Haresh et al. 2010 ). Although review papers on experimental methods and geometrical parameters are available in literature (Mavros 2001, Ascanio et al. 2004 ), a complete review on CFD applications and mixing models in reactors is not yet available (Van den Akker 2006, Ochieng et al. 2009 ), which is the focus of this review.

This review provides an insight into different approaches used in simulating multiphase fl ow and different terms of momentum balances applied in gas-liquid systems in stirred vessels. The fi rst term is momentum and stress transfer from the continuous to the dispersed phase, where different turbulence models have been investigated in this work. The second term is momentum transferred between the dispersed and the continuous phase, which depends on the force balance on each bubble and the dispersed phase distribution. Effects of drag and non-drag forces on bubbles were investigated based on force balance. The breakup and coalescence equations were investigated for distribution of dispersed phase. This review also identifi es different methods available for solving population balance equations (PBEs). As an integrated solution for various equations is required, various framework, discretization schemes, and solution algorithms are also identifi ed and analyzed. In addition, CFD simulation using mass transfer was also reviewed because results for these runs can also be analyzed from a mass transfer perspective.

\section{General approaches}

Two approaches are available for multifl uid modeling,

Eulerian-Eulerian (E-E, a two-fl uid model) and Eulerian-

Lagrangian (E-L). In the E-E multifl uid method, the continuous phase and the different dispersed phases are treated as a continuous interpenetrating media and described in terms of their volume fractions. The fl ow fi elds are solved for both phases, and the interactions between the phases are explained through the source terms (Buffo et al. 2012 ). The 
interfacial momentum transfer between liquid and gas in this model includes investigations on the infl uence of turbulent fl uctuations on the effective momentum transfer, mass force, Basset force, lift force, and the drag force (Van Wachem and Almstedt 2003 ). However, the Eulerian approach suffers from numerical diffusion from the smearing of the gas fraction over the entire grid cell (Sokolichin et al. 1997 ). In the E-L approach, the continuous phase is treated in a Eulerian framework (using averaged equations), whereas particles are tracked through the fl ow in a Lagrangian manner (Ding et al. 2010 ). A force balance is solved for each particle, and each particle is individually tracked in the system (Ashraf Ali et al. 2008 ). The second Newtonian law is also solved for each individual particle (Ashraf Ali and Pushpavanam 2011 ), and a collision model is applied to handle particle encounters (Van Wachem and Almstedt 2003 ). Generally, E-L models simulate fl ow structures with higher spatial resolution compared with E-E models and show more benefi ts for dispersed multiphase fl ows. Other benefi ts include the following:

- Reactions occurring within or on surface of individual particles, and different transport processes can be modeled in a realistic way.

- Bubble coalescence and breakup, four-way momentum exchange, and bubble-induced turbulence can be rigorously modeled.

- The exchange of momentum between the operating phases due to drag, lift, and virtual mass through the interface can be modeled more accurately in comparison with E-E.

- Dispersed size distribution can be accounted without great diffi culty (Buwa et al. 2006 ).

- Time-averaged properties could be predicted well using this viewpoint (Buwa et al. 2006 ).

However, the E-L approach faces three problems: false fl uctuations of the continuous phase velocity result in sudden changes of dispersed phase local volume fraction; the amplitude of the false velocity fl uctuation increases when a small grid is applied; as the volume fraction of dispersed phase increases, the interaction between the two phases will 
increase as well, which is not accounted for by this method

due to the considerably high demand of computational cost

(Ochieng and Onyango 2010 ); thus, this approach for a threedimensional

(3D) $\mathrm{fl}$ ow is more applicable in dilute systems

(Yeoh et al. 2005).

Full text is available at :

www.degruyter.com/view/j/revce.2012.28.issue-2-3/revce-2012-0003/revce-2012-0003.xml 